\title{
Identification et caractérisation d'un gène déterminant dans les amyotrophies spinales
}

I.es amyotrophies spinales infantiles (spinal muscular alrophy, SMA) sont des maladies neuromusculaires, caractérisées par une dégénérescence des motoneurones de la moelle épinière qui entraîne une paralysie musculaire progressive. (Ce sont les maladies héréditaires récessives autosomiques fatales les plus fréquentes après la mucovisidose (incidence $1 / 60(0)$ naissances). Aucune thérapeutique curative n'existe à ce jour.

Selon l'âge au début des signes cliniques et leur évolutivité, on distingue trois types de SMA: le type l (la forme la plus sévère de la maladie de Werdnig-Hoffmamn), le type Il (ou forme intermédiaire) et le type III (la forme la plus modérée ou maladie de Kugelberg-Welander). (he\% les enfants atteints du type l, les signes cliniques débutent clans les trois premiers mois de vie et sont caractérisés par une grande hypotonie; les nourrissons n'acquièrent pas la station assise et l'évolution est sévère du fait d'une insuffisance respiratoire gravissime. Chez les enfants atteints du type II, les signes cliniques apparaissent plus tardivement: les enfants acquièrent la station assise mais non la marche, ce qui aboutit à un handicap moteur très sévère. Les enfants atteints dı type III acquièrent la marche et, secondairement, ressentent une gêne croissante à courir puis à marcher.

L'anomalie biochimique étant incommue, la stratégie du clonage positionnel fut adoptée. La première étape consistait à localiser le gène sur une région chromosomique, puis à s'en rapprocher pour finalement l'identifier par l'observation de mutations présentes sur chacun des allèles portés par les sujets atteints et non présentes cher les sujets sains. En 1990, un groupe anglosaxon et celui de Judith Melki et Arnold Mumnich localisèrent le gène responsable des trois formes de la maladie dans la région chromosomique 5q11.2-q13.3[1-3]. Plusieurs groupes, dont le groupe parisien, entreprirent alors de se rapprocher de ce gène afïn de l'identifier. D'une distance de plus de 9cM [4] entre les marqueurs encadrant le gène, l'équipe du C.HU Necker-Enfants Malades réduisit cet intervalle à $4 \mathrm{cM}[5]$ puis à $2 \mathrm{cM}[6]$. Ces résultats permirent alors de construire un contig de chromosomes artificiels de levure (YAC:) couvrant cet intervalle à partir duquel purent être isolés de nouveaux marqueurs d'AIN très proches du gène. Cette étape révéla la présence de séquences répétées, très analogues et spécifiques de cette région. Une telle configuration de la région pouvait conférer une instabilité à l'origine de remaniements génomiques (délétions). Ainsi, une récente étude a montré l'existence de délétions de la région 5 q 13 che\% un nombre élevé de sujets atteints de la forme sévère de la maladie [7].

L'observation de délétions spécifiques che\% les malades fut un guide précieux pour cibler la recherche du gène responsable, car tout ou partie du gène se trouvait à l'évidence emporté par ces délétions [8]. Dès lors, une étude des plus petits remaniements fut entreprise, permettant un meilleur ciblage de la région candidate; parallèlement était menée la recherche de gènes, candidats par leur position dans cette région. La mise en évidence d'une large duplication inversée d'un élément d'environ 500 kpb présente sur des chromosomes normaux rendit cette étape particulièrement complexe. Néanmoins, l'identification de marqueurs recomnaissant des loci spécifiques de l'un ou l'autre de ces éléments permit de positionner les délétions des malades dans l'élément télomérique de la duplica- tion et de réduire la région critique à moins de $140 \mathrm{kpb}$. Un gène localisé dans cette région fut plus particulièrement étudié. Il est dupliqué, comme les autres marqueurs de la région, mais l'analyse comparative des séquences exoniques et des jonctions exon-intron permit de détecter cinq différences nucléotidiques entre les deux versions du gène, et donc de distinguer le gène centromérique du gène télomérique. l'analyse génétique d'une grande série d'individus sains $(n=$ 246) ou de parents de malades ( $n=$ 127) montra que le gène télomérique était toujours présent et que $95 \%$ des sujets portaient aussi le gène centromérique. ('es résutats ont suggéré que le gène télomérique était le gène ancestral et le gène centromérique sa copie. En revanche, l'analyse génétique de 229 malades de type I, II ou III montra que le gène télomérique (ancestral) était absent che\% 213 malades $(93 \%)$. De plus, che\% 13 malades $(5,6 \%)$ ce gène était interrompu entre les exons 7 et 8 . Enfin, et surtout, che\% les rares malades dont le gène télomérique n'était pas délété $(n=3)$, on observait de très petites délétions emportant les sites consensus d'épissage des introns 6 et 7, ou une mutation ponctuelle, non retrouvées chè les témoins. Ces résultats permirent de conclure que le gène télomérique dénommé SMN (pour survival molor neuron) était le gène dont les mutations sont déterminantes dans l'apparition de la maladie. Ce gène code pour une protéine de petite taille (environ 32 kDa) sans homologie avec les protéines répertoriées dans les banques de domnées. Il s'agit donc d'une protéine jusque-là inconnue et d'expression ubiquitaire [9]. I a présence d'une copie centromérique du gène, très similaire à $S M N$, amène à se demander pourquoi ce gène ne 
semble pas pallier l'absence ou les mutations du gène SMNchez les malades. A l'inverse, l'absence du gène centromérique chez les individus sains, tous porteurs du gène SMN, n'entraîne pas l'apparition de la maladie. Une différence importante de fonction semble donc exister entre le gène centromérique et SMN. L'analyse de leurs transcrits a révélé l'existence d'un épissage alternatif de l'exon 7, spécifique du gène centromérique, aboutissant à un ARNm et à une protéine plus abondants chez les malades que chez les individus sains. On peut penser que cette protéine à l'extrémité C-terminale différente de celle de la protéine SMN est non fonctionnelle ou, au moins, fonctionnellement différ ente de SMN ou du produit entier du gène centromérique.

L'absence ou l'interruption du gène SMN chez $98 \%$ des malades, quel que soit le type de la maladie, ne rend pas compte des différences cliniques qui existent entre une forme sévère et une forme modérée. Dans l'étude rapportée dans (iell[9], il est montré que chez les malades porteurs de la forme de type I, les délétions sont plus fréquentes et plus étendues que dans la forme modérée (type III). Cés délétions plus étendues pourraient emporter des éléments qui règlent l'expression de la copie centromérique analogue à $S M N$, ou emporter un autre gène proche du gène $S M N$.

Simultanément à l'équipe parisienne, Roy et al. ont identifié un gène appelé NAIP (neuronal apoptosis inhibitory protein), délété à l'état homozygote chez $17 / 38(45 \%)$ malades de type I, chez $13 / 72$ (18\%) SMA de type II ou type III, mais aussi chez $3 / 168$ (2\%) parents sains, suggérant que les mutations de ce gène sont responsables ou, plus probablement, contribuent au phénotype SMA [10]. L efebvre et al. ont localisé le gène $N A I P$ par rapport au gène SMN. NAIP, comme SMN, est dupliqué, et sa version télomérique est distale par rapport au gène SMN. Par sa position, NAIP pourrait être emporté dans les larges délétions observées chez les malades atteints de la forme sévère (type I) et non chez les malades attein ts de la forme modérée (type III). Un autre gène
(XS2(;3), identifié par Thompson et al., se trouve lui aussi être délété à l'état homozygote chez $58 \%$ des malades atteints de la forme sévère, mais aussi chez deux parents sains [11]. Cette observation semble proche de celle faite avec le gène NAIP. Des investigations plus poussées sont donc nécessaires pour confirmer l'implication d'un gène "déterminant" et de gènes "modificateurs" dans l'apparition du phénotype "amyotrophie spinale".

La capacité de détecter une anomalie moléculaire du gène SMN chez. $98 \%$ des malades permet désormais une confirmation diagnostique d'amyotrophie spinale. Aucun test biologique n'était jusqu'à présent disponible. Si la nature exacte des mécanismes génétiques qui concourent au phénotype sévère ou modéré de la maladie reste à éclaircir, l'identification du gène $S M N$, dont le rôle semble déterminant dans l'apparition de la maladie, ouvre de nombreuses perspectives. Elle permet de comnaître la protéine en cause et d'analyser sa fonction et son mode d'action dans la survie des motoneurones. Ces étapes sont des préalables essentiels pour comprendre la physiopathologie de ces maladies et définir les stratégies pour pallier cette dégénérescence motoneuronale. A cet égard, l'obtention d'un modèle animal de la maladie humaine permettrait d'envisager des essais de correction du phénotype morbide.

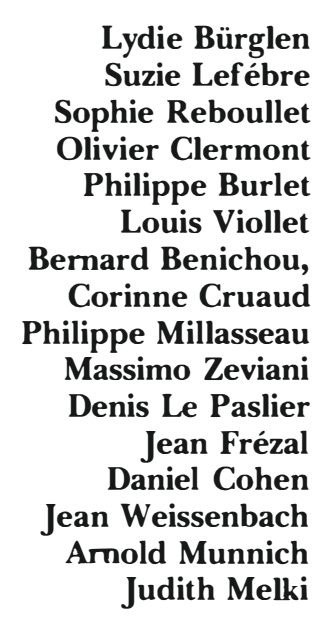

1. Brzustowicz I.M, lehner T, Castilla III, Penchaszadeh ( $K$, Wilhelmsen $K(:$, I)anicls RJ, I)dvieskE, leppert M, Ziter F, Wood D, Dubowity V, Zerres K, Hausmanova-Petrusewics 1 , () $) \mathrm{J}$, Munsat TL, (iilliam TC: (ienetic mapping of chronic childhood-onset spinal muscular atrophy to chromosome $5 q 11.2-q 13.3$. Nature 1990; 344: 540-1.

2. Melki J, Abclelhak S, Sheth P, Bachelot MF, Burlet P, Marcadel A, Aicardi J, BaroisA, Carrière JP, Fardeau $M$, Fontan D), Ponsot (; Billette $T$, Angelini (:, Barbosa (;, Ferrière (;, Lanzi (;, ()t tolini A, Babron M(:, Cohen I), Hanauer A, Clerget-I)arpoux F, I athrop M, Munnich A, Frezal J. Gene for proximal spinal muscular atrophies maps to chromosome 5q. Nature 1990) 344: 767-8. 3. Melki J, Sheth P, Abclelhak S, Burlet P, Bachelot MF, Lathrop M, Frezal J, Munnich A and the French spinal muscular atrophy investigators. Mapping of acute (type l) spinal muscular atrophy to chromosome 5 q 12-q14. L ancel 1990; 336: $271-3$.

4. Sheth P, Abdelhak S, Bachelot MF, Burlet P, Masset P, Hillaire I), ( lerget-1)arpoux F, Fréaal J, l athrop M, Munnich A, Melki J. I inkage analysis in spinal muscular atrophy by six closely flanking markers on chromosome 5. Am / Hum Genet 1991 ; 48: 764-8.

5. Melki J, Burlet P, (ilermont O, Pascal F, Paul B, Abdelhak S, Sherrington R, Gurling H, Nakamura Y, Weissenbach J, Munnich A. Refined linkage map of chromosome 5 in the region of the spinal muscular atrophy gene. Cenomics 1993; 15: $521-4$.

6. (Clermonı (), Burlet P, Bürglen I., l cefebrre S, Pascal F, McPherson J, Wasmuth IJ, (cohen I), I.e Paslier D, Weissenbach J, lathrop M, Munnich A, Melki J. Use of genetic and physical mapping (o) locate the spinal muscular atrophy locus bet ween two new highly polymorphic I)NA markers. Am J Hum (ienel 1994; 54 : 687-94.

7. Melki J, Lefebvre S, Bürglen I. Burlet P, (ilermont (), Millasseau P, Reboullet S, Bénichou B, Zeviani M, Le Paslier I), (iohen I), Weissenbach J, Munnich A. Délétions héritées et de novo de la région 5 q1.3 dans les amyotrophies spinales infantiles. medecine/sriences $1994 ; 10$ : 889-91. 8. Melki J, I ef ebvre S, Bürglen I., Burlet P, (:lermont O, Millasseau P, Reboullet S, Benichou B, Zeviani M, I.e Paslier I), (ohen I), Weissenbach J, Mumnich A. De novo and inherited deletions of the 5 q13 region in spinal muscular atrophies. Sizence 1994; $264: 1474-7$.

9. I efebvre S, Bürglen I, Reboullet S, cilermont (), Burlet P, Viollet 1.., Benichou B, Cruaud (:, Millasseau P, Zeviani M, I.e Paslier I), Frezal J, (ohen D), Weissenbach J, Munnich A, Melki J. Identilication and characterization of a spinal muscular atrophy determining gene. (iell (sous presse).

10. Roy N, Mahadevani M, Mclean M, Shutler (i, ef al. The gene for neuronal apoptosis inhibjlory protein (NAIP), a novel protein with homology to baculoviral inhibitors of apoptosis, is partially deleted in individuals with type 1,2 and 3 spinal muscular atrophy. (iell (sous presse). 11. Thompson T, Dil)onato (:, Simard 1., el al. A novel cl)NA delects homozygous microdeletions in greater than $50 \%$ of type 1 spinal muscular atrophy patients. Nature (ienet 1995 (sous presse). 


\section{Lydie Bürglen, Suzie Lefebvre, Sophie Reboullet, Olivier Clermont, Philippe Burlet, Louis Viollet, Bernard Benichou, Massimo Zeviani, Jean Frézal, Arnold Munnich, Judith Melki \\ Unité de Recherches sur les Handicaps Génétiques de l'Enfant, Inserm U. 393, Institut Necker, Hôpital des Enfants Malades, 75743 Paris Cedex 15, France.}

Corine Cruaud, Jean Weissenbach Généthon, 91002 Érry Cedex, France.

Philippe Millasseau, Denis Le Paslier, Daniel Cohen

Cientre d'Études du Polymorphisme Humain (CEPH), 27, rue Juliette Dodu, 75010 Paris, France.

\section{Remerciements}

Nous remercions les malades, les familles et les médecins qui ont contribué à la réalisation de ce projet. Nous remercions V. Raclin, N.Gigarel, M. Berthelon (Inserm U. 393) et J.F. Prudhomme (Généthon) pour leur assistance technique. Ces travaux ont bénéficié du soutien de l'Association Française contre les Myopathies (AFM), du Groupement de Recherches et d'Études sur les Génomes (GREG), la Fondation de France, la Fondation Paribas, le SESEP, le Fonds d'Études et de Recherche du Corps médical des Hôpitaux de Paris et de l'Assistance Publique, Hôpitaux de Paris. 